\title{
Advanced Ventilator Setup
}

\author{
Santosh Nadig* \\ Nursing Officer, Banaras Hindu University, Uttar Pradesh, India
}

Received: January 12, 2018; Published: January 19, 2018

*Corresponding author: Santosh Nadig, Nursing Officer, Banaras Hindu University, Varanasi, Uttar Pradesh, India, Email: santoshnadig173@gmail.com

Abbtreviations: COPD: Chronic Obstructive pulmonary disease; VT: Tidal Volume; ALI- Acute Lung Injury; WOB: Work Of Breath; CL: Lung Compliance; PEEP: Positive End Expiratory Pressure; ARDS: Acute Respiratory Distress Syndrome

\section{Introduction}

Successful mechanical ventilation requires a basic understanding of respiratory physiology and ventilator mechanics in addition to intensive nursing care. The type of breath delivered by a ventilator is determined by the combination of variables set by the operator. This combination of settings is known as a mode. The choice of appropriate ventilator settings is largely influenced by the animal's underlying disease process and usually requires some trial and error for each patient. This article reviews ventilator terminology and settings, patient setup, monitoring, and some of the common complications associated with mechanical ventilation [1].

\section{Ventilator Setups}

\section{PAV (Proportional Assist Ventilation)}

\section{A) Indications}

a. Patients who have WOB problems associated with worsening lung characteristics

b. Asynchronous patients who are stable and have an inspiratory effort

c. Ventilator-dependent patients with COPD

B) Advantages

a. The patient controls the ventilatory variables ( I, PIP, TI, TE, VT)

b. Trends the changes of ventilatory effort over time

c. When used with CPAP, inspiratory muscle work is near that of a normal subject and may decrease or prevent muscle atrophy

\section{C) Disadvantages}

a. Patient must have an adequate spontaneous respiratory b. Variable VT and/or PIP

c. Correct determination of CL and Raw is essential (difficult). Both under and over estimates of CL and Raw during ventilator setup may significantly impair proper patient-ventilator interaction, which may cause excessive assist ("Runaway") the pressure output from the ventilator can exceed the pressure needed to overcome the system impedance (CL and Raw)

d. Air leak could cause excessive assist or automatic cycling

e. Trigger effort may increase with auto PEEP

d. Lowers airway pressure

\section{PRVC (Pressure Regulated Volume Control)}

\section{A) Indications}

a. Patient who require the lowest possible pressure and a guaranteed consistent VT

b. ALI/ARDS

c. Patient with the possibility of CL or Raw changes

B) Advantages

a. Maintains a minimum PIP

b. Guaranteed VT

c. Patient has very little WOB requirement

d. Allows patient control of respiratory rate Decelerating flow waveform for improved gas distribution

e. Breath by breath analysis

C) Disdvantages

a. Varying mean airway pressure drive 

b. May cause or worsen auto PEEP
c. When patient demand is increased, pressure level may diminish when support is needed
d. May be tolerated poorly in awake non-sedated patients
e. A sudden increase in respiratory rate and demand may result in a decrease in ventilator support

\section{ASV (Adaptive Support Ventilation)}

1. A dual control mode that uses pressure ventilation (both PC and PSV) to maintain a set minimum E (volume target) using the least required settings for minimal WOB depending on the patient's condition and effort

2. It automatically adapts to patient demand by increasing or decreasing support, depending on the patient's elastic and resistive loads

3. If the patient begins having spontaneous breaths, the number of mandatory breaths decrease and the ventilator switches to PS at the same pressure level

\section{A) Indications}

a. Full or partial ventilatory support

b. Patients requiring a lowest possible PIP and a guaranteed VT

c. ALI/ARDS

d. Patients not breathing spontaneously and not triggering the ventilator

e. Patient with the possibility of work land changes (CL and Raw)

f. Facilitates weaning

B) Advantages

a. Guaranteed VT and VR

b. Minimal patient WOB

c. Ventilator adapts to the patient

d. Weaning is done automatically and continuously

e. Variable Vm to meet patient demand

f. Decelerating flow waveform for improved gas distribution

g. Breath by breath analysis

C) Disadvantages

a. Inability to recognize and adjust to changes in alveolar VD

b. Possible respiratory muscle atrophy

c. Varying mean airway pressure|

d. In patients with COPD, a longer TE may be required

e. A sudden increase in respiratory rate and demand may result in a decrease in ventilator support

\section{MMV (Mandatory Minute Ventilation)}

\section{A) Indications}

a. Any patient who is spontaneously and is deemed ready to wean

b. Patients with unstable ventilatory drive

B) Advantages

a. Full to partial ventilatory support

b. Allows spontaneous ventilation with safety net

c. Patient's E remains stable

d. Prevents hypoventilation

C) Disadvantages

a. The high rate alarm must be set low enough to alert clinician of rapid shallow breathing

b. Variable mean airway pressure

c. An inadequate set E (>spontaneous E) can lead to inadequate support and patient fatigue

d. An excessive set E (>spontaneous E) with no spontaneous breathing can lead to total support

\section{APRV (Airway Pressure Release Ventilation)}

\section{A) Indications}

a. Partial to full ventilatory support

b. Patients with ALI/ARDS

c. Patients with refractory hypoxemia due to collapsed alveoli

d. Patients with massive atelectasis

e. May use with mild or no lung disease

B) Advantages

a. Allows inverse ratio ventilation (IRV) with or without spontaneous breathing (less need for sedation or paralysis)

b. Improves patient-ventilator synchrony if spontaneous breathing is present

c. Improves mean airway pressure

d. Improves oxygenation by stabilizing collapsed alveoli

e. Allows patients to breath spontaneously while continuing lung recruitment

f. May decrease physiologic deadspace

\section{C) Disadvantages}

a. Variable VT

b. Could be harmful to patients with high expiratory resistance (i.e., COPD or asthma) 


\section{c. Auto-PEEP is usually present}

d. Caution should be used with hemodynamically unstable patients

e. Asynchrony can occur is spontaneous breaths are out of sync with release time

f. Requires the presence of an "active exhalation valve"

\section{Conclusion}

Successful ventilation requires a basic understanding of respiratory physiology and ventilator mechanics in addition to extensive nursing care. Mechanical ventilation can allow stabilization and maintenance of patients in which other less invasive therapeutic options have been exhausted. PPV is an essential component of human intensive care and will inevitably become the advanced standard of respiratory care in veterinary medicine as veterinarians gain more experience with this modality.

\section{References}

1. Bransonrd (2001) campbellrs: modes of ventilator operation, in macintyre nr, bransonrd (Eds.) mechanical ventilation. wbsaunders, philadelphia, USA, p. 51-84.

\section{(C) (i) This work is licensed under Creative} Submission Link: http://biomedres.us/submit-manuscript.php

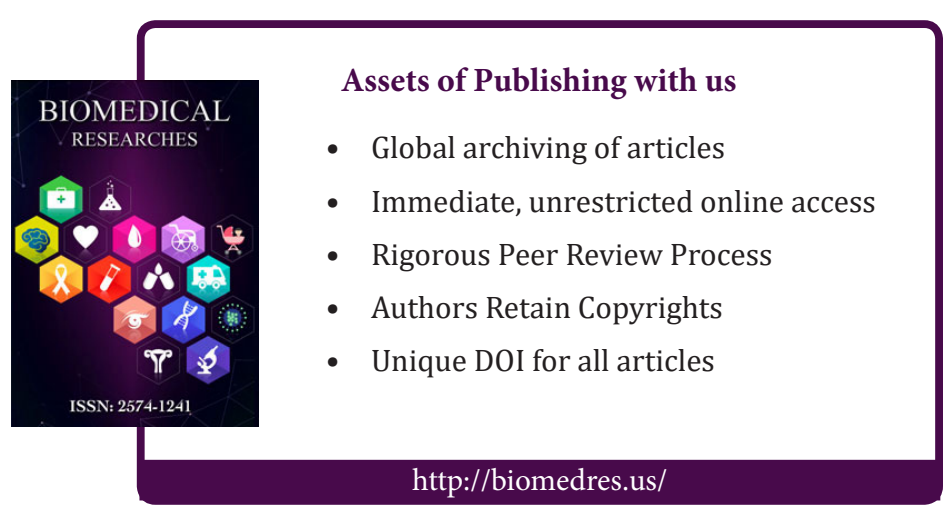

